Revue d'histoire de l'Amérique française

ZWB REVUE D.HISTOIRE DE L'AMÉRIQUE FRANÇAISE

\title{
Colonisation et rapatriement au Lac-Saint-Jean (1895-1905)
}

\section{Robert G. LeBlanc}

Volume 38, numéro 3, hiver 1985

Population et histoire

URI : https://id.erudit.org/iderudit/304284ar

DOI : https://doi.org/10.7202/304284ar

Aller au sommaire du numéro

\section{Éditeur(s)}

Institut d'histoire de l'Amérique française

\section{ISSN}

0035-2357 (imprimé)

1492-1383 (numérique)

Découvrir la revue

\section{Citer cet article}

LeBlanc, R. G. (1985). Colonisation et rapatriement au Lac-Saint-Jean (1895-1905). Revue d'histoire de l'Amérique française, 38(3), 379-408. https://doi.org/10.7202/304284ar d'utilisation que vous pouvez consulter en ligne.

https://apropos.erudit.org/fr/usagers/politique-dutilisation/ 


\section{COLONISATION ET RAPATRIEMENT AU LAC-SAINT-JEAN $(1895$ - 1905)*}

ROBERT G. LEBLANC

Département de géographie

Université du New Hampshire

\section{Introduction}

Entre 1840 et 1940, plus d'un million de Canadiens français quittèrent leur terre natale de la vallée du Saint-Laurent pour aller s'établir dans les centres industriels de la Nouvelle-Angleterre et du Midwest américain ${ }^{1}$. Dès 1870 , ce mouvement migratoire avait atteint des proportions dont s'inquiétaient à juste titre les dirigeants tant laïques que cléricaux de la province de Québec. Pour certains, un tel exode représentait une menace à la survie de la culture française en Amérique du Nord. En effet, toute diminution sensible de la population francophone du Canada pouvait signifier une réduction éventuelle du pouvoir politique de ce groupe au sein de la nouvelle Confédération. Le mouvement de colonisation lancé vers le milieu du siècle avait pour but d'étendre l'agriculture vers les terres peu exploitées de la province; c'était une solution au surplus de la population des terres basses, population qui eût été obligée d'émigrer ailleurs. Comme suite logique à la colonisation, l'on mit sur pied un mouvement de rapatriement des Canadiens

* Une version préliminaire de cet article fut présentée à la réunion annuelle de l'Association des géographes canadiens en mai 1983 à Winnipeg. Je remercie mon collègue W. H. Wallace pour ses critiques pertinentes. La recherche fut subventionnée par le Central University Research Funds de l'Université du New Hampshire et par une bourse de recherche du Gouvernement du Québec.

I Il existe un vaste inventaire de publications sur les migrations, pour la plupart répertoriées dans les archives du Québec ou d'Ottawa. Parmi les travaux récents à valeur statistique et critique, on trouve celui de Ralph Vicero «The Immigration of French-Canadians to New England. 18401900», thèse de Ph. D., Université du Wisconsin, 1968. Voir aussi deux études de Yolande Lavoie, L'émigration des Canadiens aux États-Unis avant 1930 (Les Presses de l'Université de Montréal, 1972) et «Les mouvements migratoires des Canadiens entre leur pays et les États-Unis aux XIX ${ }^{\mathrm{e}}$ et $\mathrm{XX}^{\mathrm{e}}$ siècles: étude quantitative», dans Hubert Charbonneau, La population du Québec: études perspectives (Montréal, Boréal Express, 1973), 73-88; Gilles Paquet «L'émigration des Canadiens français vers la Nouvelle-Angleterre, 1870-1910, prises de vue quantitatives», Recherches sociographiques, 5 (1964): 319-370; Albert Faucher, «L'émigration des Canadiens français au XIX ${ }^{\mathrm{e}}$ siècle: position du problème et perspectives». Pour une interprétation originale de la diaspora canadienne-française, voir Christian Morissonneau, «Mobilité et identité québécoise», Cahiers de géographie du Québec, 23 (1979): 29-38. 
français qui s'étaient déjà établis aux États-Unis. Il faut donc considérer colonisation et rapatriement comme les mouvements complémentaires d'une seule démarche, dont le but était de provoquer l'essor de la culture canadienne-française dans la vallée du Saint-Laurent ${ }^{2}$.

Cette démarche reçut l'appui de bon nombre d'individus et d'institutions en place. L'éminent curé Labelle l'encouragea avec enthousiasme; les gouvernements fédéral et provincial s'y impliquèrent à maintes occasions. Plusieurs sociétés furent fondées tant aux États-Unis qu'au Québec pour promouvoir cette cause. Bon nombre d'entre elles étaient de petite envergure, financièrement faibles, mal administrées, ne servant tout au plus qu'à rehausser le prestige patriotique de leur fondateur; elles n'eurent pas longue vie. En revanche, les sociétés plus importantes et plus solides au niveau du leadership et des ressources financières s'imposèrent pendant longtemps comme promotrices de la démarche. Des docurnents relatifs à l'une d'elles, la Société de rapatriement et de colonisation du Lac-Saint-Jean, nous apprennent que colonisation et rapatriement n'évoluèrent pas sans difficulté. Par exemple, en dépit des aspirations communes des deux groupes impliqués, il y eut conflit entre les dirigeants de la société et les fonctionnaires fédéraux à propos des politiques d'immigration et de financement. Dès le départ, surgirent des discordes quant aux modes de financement de la société, et ce sont ces mêmes conflits qui plus tard provoquèrent la dissolution de l'organisme.

Notre étude s'attardera principalement à évaluer les différents éléments qui caractérisaient les émigrants en puissance, établis dans des régions particulières, que nous appellerons les «zones migratoires» ${ }^{3}$. Certaines des estimations de départ s'avérèrent correctes alors que d'autres durent être revisées à la lumière de données subséquentes. Il

2 Il existe une littérature abondante concernant le mouvement de colonisation. Un consultera avec profit les rapports publiés par le gouvernement du Québec et les publications, sous forme de documents de propagande, d'organismes nationalistes ou religieux. Comme exemple d'étude détaillée, voir Normand Séguin, La conquête du sol au $19^{e}$ siècle (Boréal Express, 1977), dont l'objet particulier est la colonisation à Hébertville. Voir aussi la récente étude explicative de Christian Morissonneau, La terre promise: le mythe du Nord québécois (Hurtubise HMH, 1978). Par contre, le mouvement de rapatriement n'a pas encore été aussi bien scruté par les historiens. Ce n'est que circonstanciellement qu'il a reçu une certaine attention de ceux qui ont étudié l'immigration (voir la note précéclente). Robert Painchaud consacre un chapitre au rapatriement dans sa thèse, «The Catholic Church and the Movement of Francophones to the Canadian Prairies, 1870-1915» (Thèse de $\mathrm{Ph}$. D., Université d'Ottawa, 1976). On trouve un chapitre sur le rapatriement dans Norman MacDonald, Canada: Immigration and Colonization, 1841-1903 (Toronto, MacMillan of Canada, 1968). Pour d'autres travaux récents, voir Donald Chaput, «Some Repatriement Dilemmas», Canadian Historical Review, 49 (1968): 400-412; Robert G. LeBlanc, «Regional Competition for Franco-American Repatriates», Quebec Studies, 1 (1983): 110-129; John I. Little, «La Patrie: Quebec's Repatriation Colony, 1875-1880», communication présentée en juin 1977 au congrès annuel de la Société historique du Canada.

Hägerstrand définit une zone migratoire comme «la répartition des migrants telle que vue à partir de la paroisse ou de la commune observée». Cette définition s'applique à la présente étude. Torsten Hägerstrand, «Migration and Area» dans Migration in Sweden: A Symposium, Lund Studies in Geography Series B, Human Geography 13 (1957): 29. 
faut aussi inclure dans le tableau de la migration des éléments tels que l'agrément du projet par le Fédéral, la présence possible au sein d'une société, de conflits d'intérêts entre ses objectifs économiques et ses objectifs patriotiques et, en dernier lieu, la rivalité entre plusieurs régions canadiennes, chacune tentant de maintenir sa communauté francophone en y attirant de nouvelles familles.

La Société de rapatriement du Lac-Saint-Jean vit le jour en 1897. Elle se distingua des sociétés qui l'avaient précédée par sa structure imposante, ses assises financières solides et le fait que son objectif de rapatriement était clairement énoncé ${ }^{4}$. Elle naquit d'une conjoncture de facteurs qui laissaient croire, vers les années 1890, au développement économique de la région du Saguenay ${ }^{5}$. Jusque vers le milieu du XIX siècle, le développement du Saguenay avait été sérieusement handicapé par le monopole de la traite des fourrures ${ }^{6}$. Après 1850 , les activités de William Price et de quelques petits entrepreneurs canadiens-français donnèrent à l'industrie du bois un essor considérable. La colonisation agricole commença, à la même époque, et connut une croissance modeste. En 1891, on comptait moins de 4000 fermes et la plupart des terres arables étaient encore boisées ${ }^{7}$. L'isolement représentait l'obstacle majeur à l'expansion de l'exploitation forestière et au développement de l'agriculture commerciale. La route maritime Saguenay-SaintLaurent était fermée par les longs et durs hivers; les communications par terre étaient plus que rudimentaires. On finit par construire deux chemins pour chariots, ce qui atténua quelque peu l'isolement hivernal. Toutefois, ces chemins étaient impraticables au printemps et au début de l'été. La situation s'améliora grandement quand fut complété le dernier tronçon du chemin de fer Québec-Lac-Saint-Jean. L'arrivée de la voie ferrée à Chicoutimi en 1893 redonna espoir aux promoteurs de la colonisation et raviva leurs discours enthousiastes sur le potentiel économique de la région. Leur optimisme allait même jusqu'à évoquer le jour où le Saguenay serait le grenier de l'Est, à l'instar des Prairies pour l'Ouest, et Chicoutimi, le New York canadien ${ }^{8}$.

4 Normand Séguin, op. cit., chapitre III. On y relate l'échec de plusieurs associations ou sociétés qui tentèrent d'attirer des colons à Hébertville entre 1850 et 1870.

5 Pour nous, la région du Saguenay comprend les rives du Saguenay et les terres basses adjacentes au lac Saint-Jean. Il s'agit d'un seul bassin de terres, mais chaque partie possède ses caractéristiques propres. Le Haut-Saguenay, en particulier la région entourant Chicoutimi, possède d'énormes ressources hydrauliques mais peu de terres arables. Par contre, les basses terres autour du lac Saint-Jean sont pauvres en ressources hydrauliques mais ont une origine et une qualité comparables à celles du Saint-Laurent.

6 Le tableau des grandes lignes de l'économie tracé ici est basé sur l'ouvrage de William F. Ryan, The Clergy and Economic Growth of Quebec 1896-1914 (Québec, Les Presses de l'Université Laval, 1966). Cette étude relate l'influence du clergé et de l'Église catholique comme promoteurs plutôt inattendus du développement économique. Le chapitre IV traite de ces activités dans la région du Saguenay et comporte un bref historique.

7 Voir données et cartes dans Séguin, op. cit., 52-53, 65, 67. Les cartes sont tirées de C.M. Johnston, «The Historical Geography of the Saguenay Valley», thèse de maîtrise, Université McGill, 1950.

8 Ryan, op. cit., 152; Morissonneau, op. cit., 135, 183. 
Il semble toutefois que les tendances de l'économie continentale et mondiale ne justifiaient pas tout à fait cette exubérance. Dans un monde où les marchés étaient en pleine dépression, il était peu probable que les produits de l'agriculture et de l'industrie forestière puissent trouver des débouchés intéressants. Néanmoins, la crise économique des années 1890 alimenta l'espoir d'un développement agricole des terres basses du Lac-Saint-Jean. On présumait que la crise entraînerait la fermeture des filatures de coton en Nouvelle-Angleterre, ce qui contribuerait à réduire le taux d'émigration et à favoriser un certain mouvement de rapatriement, comme cela s'était passé pendant les années $1870^{9}$. On espérait ainsi que la crise économique garantirait le triomphe des promoteurs et se concrétiserait par la venue d'un flot régulier de défricheurs au Lac-Saint-Jean. Ces défricheurs seraient des fils des terres basses du Saint-Laurent que la Nouvelle-Angleterre n'attirerait plus. On espérait aussi la venue de Franco-Américains de Manchester, Fall River, Woonsocket et Holyoke. Comme des enfants prodigues fuyant la misère, ces citadins expatriés reviendraient à la mère patrie pour y vivre une vie plus humaine.

Finalement, l'accession au pouvoir du Parti libéral en 1896 redonna espoir à l'élite québécoise qui attendait toujours plus du gouvernement en faveur du rapatriement et de la colonisation. Les Canadiens français allaient enfin pouvoir négocier directement avec Ottawa puisque, pour la première fois depuis la Confédération, l'un des leurs détenait le plus haut poste du pays. Wilfrid Laurier n'aurait peut-être pas le zèle inconditionnel des nationalistes comme Henri Bourassa, mais même les conservateurs québécois pouvaient s'attendre à recevoir auprès de lui un accueil sympathique. À peine fut-il installé à Ottawa que commencèrent à pleuvoir les suggestions et les demandes de subventions. Dans une lettre de félicitations au Premier ministre en date du 1 er septembre 1896, messieurs Johnston et Daly, de la Chenango Mutual Relief d'Oxford, New York, expriment l'opinion suivante:

A great many of your countrymen who are at present located in American border cities have, on account of the change in government in Canada, a longing desire to once more return to their native land... The present time is an auspicious one to encourage immigration from the northern part of the United States... the universal cry here is "hard times, no work, and no money».

9 On admettait, de manière générale, qu'il existait une relation entre crise économique et possibilités de succès du rapatriement; ce qui amena le gouvernement à expédier des agents dans les villes où les fermetures d'usines avaient été récemment effectuées, ou même seulement pressenties. Voir, par exemple, Québec, Documents de la session, Rapports annuels d'agriculture et de Colonisation des années 1895, 1900, 1905, 1914 et Canada: Sessional Papers, Annual Reports for Immigration for the years 1894, 1897, 1903 
Ces représentants affirmaient qu'une aide financière aurait pour effet d'amener un «large influx of people» ${ }^{10}$. Dans une démarche similaire, le docteur Moïse Guérin, à titre de président d'une nouvelle société, le Club de rapatriement de Manchester (New Hampshire), présenta une requête de financement, soutenant que «s'il était encouragé comme il le mérite, le Club de Manchester verrait bientôt rayonner son oeuvre dans toute l'étendue de la Nouvelle-Angleterre ${ }^{11}$. Laurier était en mesure de prêter une oreille sympathique à ces demandes puisque le rapatriement était compatible avec la politique d'immigration du gouvernement. Mais le Premier ministre et le ministère de l'Immigration furent souvent en désaccord avec les promoteurs de la province de Québec, dont plusieurs étaient des adversaires politiques.

\section{Formation de la Société de rapatriement}

La Société de rapatriement fut fondée en 1896, inspirée, d'une part, par les objectifs commerciaux de la compagnie Quebec-Lake St. John Railway et, d'autre part, par les ambitions patriotiques du clergé et autres défenseurs de la colonisation et du rapatriement. Née dans la controverse, elle devait être dissoute onze ans plus tard au milieu de conflits similaires. Les premières démarches en vue de l'obtention de subsides du gouvernement furent entreprises quelques jours avant l'élection qui porta Laurier au pouvoir. Au nom de la Compagnie de chemin de fer, le député L. de G. Belley adressa au Ministre de l'intérieur T. Mayne Daly une demande de subvention au montant de $14000 \$$ par an, pour cinq ans, affirmant que ledit chemin de fer constituait une «route de colonisation».

The Company would agree, in consideration of this grant, to organize a movement of immigration for repatriation of FrenchCanadians from the United States and their settlement in the Lake St. John region. Moreover, the Company would agree to carry the colonists and their effects free of charge over the line to Lake St. John as it has already done in the past and also to provide refrigeration cars on its lines for the transportation of dairy products, from Lake St. John and Chicoutimi. ${ }^{12}$

La demande fut rejetée. On estimait que les sommes demandées étaient exorbitantes et on croyait que la demande aurait dû être soumise soit au gouvernement provincial, davantage impliqué dans le mouvement de colonisation, soit au ministère de l'Agriculture, intéressé à développer le marché des produits laitiers ${ }^{13}$. Deux semaines plus tard, les conservateurs étaient défaits.

\footnotetext{
10 Archives publiques du Canada (ci-après désignées APC) Laurier Papers, 6855.

11 APC, Ibid., 19063-19064.

12 Lettre de L. de G. Belley à T. Mayne Daly, 14 avril 1896, APC, RG-76, Immigration, vol. 129 , file 28703 .

13 Lettre de A. M. Burgess à T. Mayne Daly, 24 avril 1896, Ibid.
} 
La Compagnie décida de réitérer sa demande au nouveau gouvernement qu'on présumait beaucoup plus sympathique à l'immigration. La requête adressée par J.E. Scott, secrétaire de la Compagnie, reçut en effet un bon accueil de la part de Laurier qui répondit: «I am authorized to say the grant will be made good ${ }^{14}$, même si les sommes impliquées n'étaient pas encore disponibles. Scott semblait pressé, et moins de trois mois plus tard, il revenait à la charge auprès de Laurier, cette fois réclamant carrément un octroi de $8000 \$$ par an pendant vingt ans, «as was done in the case of the railways in the western territories» ${ }^{15}$. Il appuya sa demande en présentant un rapport sur les travaux de colonisation déjà accomplis par sa Compagnie. Une seconde lettre fut adressée presque en même temps au Ministre de l'intérieur, Clifford Sifton:

I understand it is the intention of the Government to devote a portion of the money formerly applied for immigration purposes to assist colonization societies... I trust that we may have your sympathy and assistance in the good work which we are doing in this direction. ${ }^{16}$

Ces lettres donnent une bonne idée de la stratégie sur laquelle voulait s'appuyer la Compagnie pour obtenir des subsides d'Ottawa: leur chemin de fer méritait tout autant d'être subventionné que ceux de l'Ouest, qui, en fait, avaient joui de fonds en provenance des impôts québécois, du moins en partie. De plus, on prétendait qu'un organisme au service du rapatriement, et en conséquence contre l'émigration, avait des objectifs tout à fait conformes aux politiques du ministère de l'Immigration qui visait à accroître la population du Canada. La Société devait donc pouvoir espérer recevoir des fonds comparables à ceux qui seraient versés à un bureau d'immigration situé à Londres ou à Liverpool.

Les espoirs de la Compagnie ne furent pas déçus. Impressionné par les représentations de Scott, Sifton appuya les demandes de la Compagnie auprès du gouverneur général:

Under the circumstances, the Company submits that they are entitled to receive assistance at the hands of the Dominion Government as the work which they are prosecuting is of a national character and one of the most potent means to check the expatriation of Canadians to the United States. ${ }^{17}$

Sifton recommanda un octroi de $8000 \$$, mais le vérificateur général, J.L. McDougall, s'y opposa. Ce fonctionnaire de carrière fit valoir ses arguments auprès de J.A. Smart, sous-ministre de l'intérieur:

\footnotetext{
14 Lettre de Laurier à J.G. Scott, 19 janvier 1897, Ibid .

15 Lettre de J.G. Scott à Laurier, 4 avril 1897, Ibid.

16 Lettre de J.G. Scott à Sifton, 7 avril 1897, Ibid.

17 Lettre de Sifton au Gouverneur général, sans date, Ibid.
} 
It does not appear that there was any understanding with the Government that the R.R. Co. should be reimbursed for its expenses nor is there any evidence of what the expense was, nor whether the settlers were mainly from other parts of Canada or from the U.S.... It would lead to great difficulties if Companies or individuals could just make expenditure... and have ground for hoping to have claims for reimbursement granted. ${ }^{18}$

La controverse fit rage encore cinq mois entre le ministère de l'Intérieur et le bureau du Vérificateur général, principalement sous la forme d'une correspondance acerbe entre un sous-ministre imbu de son importance et un bureaucrate exaspéré ( $« \mathrm{I}$ have been here many years and you have been in office only a few months»). Le problème était de savoir si le gouvernement pouvait et devait allouer un tel octroi. Smart exposa clairement la nouvelle politique d'immigration du gouvernement:

In view of the fact that for the service for which their grant is being made to the Company, the Government in many other ways has spent large sums of money, namely in the repatriation of Canadians and in general immigration work. I do not see that... there should be any difference as the real object to be served is the settlement of the wastelands of Canada (sic)... and this Company has certainly shown its ability to successfully do this work. ${ }^{19}$

Le conflit prit fin, semble-t-il, en novembre 1897, lorsque le Conseil du trésor, sur avis du Ministre de la justice, se prononça contre l'opinion du vérificateur général ${ }^{20}$. Entre-temps, Smart avait tout bonnement accordé l'octroi et expédié un chèque de $8000 \$$ à la Compagnie ${ }^{21}$.

C'est au beau milieu de cette période de controverse que fut mise sur pied la Société de rapatriement et de colonisation qui, toutefois, ne fut subventionnée par Ottawa qu'en 1901. Elle reçut tout de suite l'appui inconditionnel de la Compagnie de chemin de fer et de l'élite du Canada français. Si ces deux groupes étaient d'accord, c'est qu'ils reconnaissaient que les objectifs de la Société concouraient effectivement au développement des secteurs rural et urbain de l'économie du Saguenay. Un texte récent de Brian J. Young exprime l'avis que le clergé était assez vulnérable face aux hommes d'affaires à cause justement de son appui enthousiaste au chemin de fer.

As all levels, from archibishop to curé, the Church joined in the exhileration for railways, industrial capitalism and the new technology. Entrepreneurs capitalized on these susceptibilities by inviting priests into the boardrooms, by putting French-Canadian

\footnotetext{
8 Lettre de J.C. McDougall à J.A. Smart, 3 juin 1897, Ibid.

Lettre de Smart à McDougall, 7 juillet 1897, Ibid.

Procès-verbal des réunions du Conseil du Trésor, 20 novembre 1897, ibid.

Lettre de Smart à J.G. Scott, 28 juillet 1897.
} 
lawyers in their front offices and by labeling their railways as colonization projects. ${ }^{22}$

Si l'on admet que l'élite canadienne-française ne cherchait qu'à réaliser ses objectifs patriotiques, il faut cependant se demander si l'appui des compagnies de chemin de fer n'était pas qu'une manoeuvre et ne déguisait pas simplement leurs objectifs commerciaux réels.

\section{Les objectifs de la Société de rapatriement}

Ces objectifs sont clairement énoncés par le secrétaire de la Société, René Dupont, dans une lettre adressée à Sifton.

La Société de rapatriement was formed with the object of promoting immigration and colonization to the Lake St. John district and North as far as Hudson Bay. Our aim is to have immigrants and our expatriated Canadians to go and settle there. (sic) ${ }^{23}$

En vérité, étendre la colonisation vers le nord n'avait aucune signification puisqu'il n'existait aucune route au nord du Lac-Saint-Jean. Cette constatation ne fait que refléter l'idéologie expansionniste et l'optimisme sans bornes de ceux qui prétendaient guider la destinée des Canadiens français ${ }^{24}$. Même si le Saguenay était devenu tout à fait accessible, il se trouvait encore des gens pour réclamer une extension de la voie ferrée jusqu'à la baie d'Hudson ${ }^{25}$.

Dès le moment de sa fondation, la Société fut confrontée au sein même de son organisation à des contradictions qui l'obligeaient à servir deux maîtres à la fois, pour ainsi dire. D'une part, elle se devait de veiller aux intérêts économiques du Québec-Lake St. John Railway qui l'avait créée. D'autre part, comme société de colonisation et de rapatriement, son devoir était avant tout «nationaliste». Dans le cadre de son premier mandat, elle devait assurer la venue régulière de colons vigoureux, prêts à défricher la terre et à construire des fermes. Elle devait encourager un système agricole de plus en plus spécialisé et orienté vers le commerce. Il n'était pas question de se limiter à une agriculture de subsistance puisque le chemin de fer pouvait exporter une production devenue commerciale. De plus, il fallait former un grand

\footnotetext{
22 Brian J. Young, Promoters and Politicians: The North Shore Railways in the History of Quebec, 1854-85 (Toronto, University of Toronto Press, 1978), 143.

23. Lettre de Dupont à Sifton, 19 novembre 1897, APC, RG-76, Immigration, vol. 129, file 28703, part 1. Cette lettre et d'autres de Dupont laissent croire que lorsqu'il parle de Canadiens expatriés, il veut dire les Canadiens français du Québec.

${ }_{24}$ Morissonneau, op. cit., Paul Claval, «Le Québec et les idéologies territoriales», Cahiers de Géographie du Québec, 24 (1980): 31 -46. Voir aussi, La Société de Colonisation du Lac-SaintJean, Terres à Blé et Industries du Nord de Québec, $2^{\mathrm{e}}$ édition (Québec, Dussault et Proulx, 1905), 57-58.

25 Morissonneau, op. cit., 183.
} 
nombre de travailleurs spécialisés dans l'industrie du bois, l'une des industries «pionnières» du Saguenay.

Quant aux objectifs patriotiques et nationalistes de la Société, ils pourraient être atteints précisément en suivant l'exemple des sociétés de rapatriement et de colonisation qui, pendant cinquante ans, avaient toujours suivi la même ligne de conduité: offrir aux émigrants éventuels ainsi qu'aux Canadiens «exilés» aux États-Unis un milieu de vie qui leur serait plus agréable au Québec ou dans toute autre région du Canada. L'atteinte de cet objectif garantirait la survie de la culture canadiennefrançaise au Québec. On espérait que la population francophone allait s'étendre vers le nord et l'ouest, que sa croissance serait telle par rapport aux autres groupes ethniques en présence qu'il resterait peu de chance qu'elle fût assimilée par l'Amérique anglophone. Il semblait d'ailleurs facile d'atteindre les deux objectifs, à la condition, bien sûr, que les nouveaux colons fussent des Canadiens français ou des FrancoAméricains ou encore des immigrants de l'Europe francophone. Il est vrai que la venue d'autres groupes ethniques non francophones aurait pu être bénéfique au développement économique, mais elle aurait constitué une menace pour la survie de la francophonie.

La lettre de Dupont, citée plus haut, fait état d'une politique qui n'impose aucune restriction quant à l'ethnie des futurs colons du Saguenay. Certes les Canadiens français et les Franco-Américains représentaient le groupe-cible, mais on n'écartait pas pour autant la possibilité d'aller chercher des immigrants ailleurs en Amérique ou en Europe. La Société ne se montra pas indifférente à l'origine des nouveaux arrivants, comme on le verra plus loin, mais après plusieurs années d'opération fructueuse au Québec et en Nouvelle-Angleterre, elle se tourna finalement vers l'Europe comme source de recrutement.

\section{Stratégie pour attirer les immigrants}

Les méthodes utilisées par la Société pour encourager l'immigration au Haut-Saguenay ne furent pas différentes de celles qu'on a généralement employées pour attirer les immigrants vers des terres nouvelles destinées à l'agriculture. La Société et le ministère fédéral de l'Immigration préparèrent, chacun de leur côté ${ }^{26}$, une documentation en plusieurs langues. De manière générale, on peut qualifier cette propagande de trompeuse, car elle s'appliquait à montrer les avantages de la région tout en passant délibérément sous silence les difficultés de coloniser une terre vierge. Les auteurs y manifestent un zèle intempestif à vouloir recruter le plus grand nombre possible d'immigrants, et une très

\footnotetext{
26 Depuis 1867 , les deux gouvernements avaient juridiction sur l'immigration, ce qui ne fut pas sans amener des conflits et une certaine concurrence.
} 
grande naïveté dans la description des lieux ${ }^{27}$. La documentation était distribuée sur demande par la poste, et de main à main par des représentants de la Société et du gouvernement fédéral à des endroits jugés stratégiques.

Une autre méthode de distribution consistait à faire appel à des représentants itinérants engagés et payés directement par la Société ou par le gouvernement fédéral. C'étaient parfois des laïcs mais le plus souvent des membres du clergé. Ces «missionnaires colonisateurs» étaient choisis pour leur pouvoir de persuasion et pour la haute estime dont ils jouissaient auprès des Canadiens français et des FrancoAméricains. Comme ils ne parlaient que le français, leur «champ de mission» se limitait à la population francophone du Québec et des ÉtatsUnis. Ils firent la tournée des vieilles paroisses de la vallée du St-Laurent et des principaux centres industriels franco-américains. Leur stratégie consistait à rallier à leur cause les membres du clergé, les éditeurs de journaux et les autres membres de l'élite locale. Leur influence était d'autant plus appréciable qu'ils jouissaient déjà, comme hommes d'Église, d'une bonne crédibilité auprès du public. Ils présentaient des conférences enthousiastes, accompagnées de la projection de plaques de lanterne magique montrant, par exemple, des colons heureux, vivant au milieu de champs de céréales florissants, sous la protection sereine des clochers rustiques. Le scénario comportait aussi la lecture de témoignages favorables venant de colons déjà établis. Le «missionnaire» faisait ensuite appel au patriotisme et à l'esprit religieux de l'assistance. Aux habitants du Québec, on faisait valoir que la colonisation du Saguenay constituait un moyen de salut autant personnel que national car, soutenait-on, si l'émigration se faisait vers le monde anglo-saxon, cela pouvait signer l'arrêt de mort de leur culture, de leur langue et de leur religion. Aux auditoires franco-américains, on adressait un discours destiné à susciter la douce nostalgie de la mère patrie: ne seraitil pas merveilleux de retourner à une vie meilleure au Canada, de racheter du coup l'erreur de l'expatriation, et de contribuer ainsi à la survie de la culture française en terre d'Amérique?

À l'intention des futurs colons, la Société organisa des visites au Lac-Saint-Jean. La plupart des délégations venaient des vieilles paroisses du Québec et se composaient de deux personnes, dont habituellement le curé. Quelques représentants franco-américains vinrent également

27 Par exemple, une des premières techniques de propagande consistait à insinuer que les terres du nord de l'Amérique possédaient un climat aussi clément et étaient aussi productives que d'autres régions du monde situées sous les mêmes latitudes, telles «les $48^{\mathrm{e}}$ degrés de latitude nord, qui sont absolument les mêmes que ceux sous lesquels se trouvent situés les endroits les plus privilégiés de France, c'est-à-dire dans les environs de Paris». Terres à Blé et Industries du Nord de Québec (Dussault et Proulx, 1905), $2^{\mathrm{e}}$ édition, 10. Pour une analyse récente du vaste inventaire de propagande agraire au Québec, voir Lois M. Rock, «The Agrarian Propaganda of Nineteenth Century French Canada», Thèse de Ph. D., Université de Birmingham (Angleterre), 1979. 
constater de visu l'état des choses. Lorsque ces visites s'avéraient fructueuses, on présentait une demande en bonne et due forme, et la Société émettait des billets certifiant aux futurs colons l'allocation d'une terre et le transport gratuit.

\section{Les zones de migration}

Le tableau qui suit indique les origines géographiques des nouveaux colons du Lac-Saint-Jean. Au cours de sa brève existence, la Société de rapatriement paya des frais de voyage à plusieurs centaines de délégations et émit 18658 billets de colonisation. Les données indiquent que la majeure partie des arrivants étaient du Québec et de la Nouvelle-Angleterre, presque tous des francophones. On compte très peu de colons venant d'autres provinces que le Québec. Les quelques colons en provenance de l'Ontario originaient de la vallée de l'Outaouais, de Windsor et de Sudbury, toutes des régions présumément francophones. Ceux des Maritimes étaient des Acadiens, presque tous originaires de Cheticamp en Nouvelle-Écosse. Parmi les émigrants américains, outre ceux de la Nouvelle-Angleterre, on en retrouve de Patterson au New Jersey, de Détroit, de Chicago et de plusieurs villes du Centre-Nord où l'on exploitait le bois et les mines et où s'étaient expatriés des Canadiens français. D'ailleurs, nous constaterons plus loin que tous ces nouveaux colons «américains» n'étaient en fait que d'anciens Canadiens français.

De l'extérieur du continent, il vint peu d'immigrants et, comme on pouvait s'y attendre, c'est l'Europe qui fournit le plus gros de l'immigration non francophone. Le recrutement de francophones de France et de Belgique fut assez substantiel. Mais en 1902-1903, arriva un vaste effectif de colons, en provenance du nord de l'Europe, soit environ $10 \%$ du total des immigrants. Certes, en s'intéressant à ce type d'immigrants, la Société s'écartait de sa ligne de conduite qui consistait à attirer exclusivement des immigrants francophones. Il ne vint qu'un très petit nombre d'allophones, mais il fallut tout de même justifier leur présence. Même si l'arrivée de colons costauds était bénéfique la venue de non-francophones comportait cependant le dilemme, ou de leur assimilation, ou d'une diminution éventuelle du pouvoir politique des francophones au Québec ou au Canada.

On peut également conclure que le nombre de colons en provenance d'une zone migratoire donnée représente une certaine mesure de l'efficacité des stratégies de promotion en ce lieu. Les émigrants en puissance, fussent-ils d'Europe, des États-Unis ou du Canada, disposaient de bien d'autres choix que le Lac-Saint-Jean. On constate que la Société a fait preuve de prudence et de sagesse dans ses décisions. Par exemple, elle a dû bien identifier ses groupes-cibles et bien établir ses critères de sélection qualitatifs et quantitatifs. 


\section{Critères de sélection}

\section{Les Franco-Américains}

Ce groupe fut considéré, dès le départ, comme une cible de choix. La plupart étaient des Québécois récemment expatriés, installés dans des localités de la Nouvelle-Angleterre, peu éloignées du Québec natal et facilement accessibles par chemin de fer. Il était notoire que ces citoyens avaient quitté leur pays avec la ferme intention d'y revenir après avoir amassé un petit pécule leur permettant de se payer l'achat d'une ferme ${ }^{28}$. Ceux qui, à l'époque, voyaient d'un mauvais oeil une telle émigration, ne se gênaient pas pour prédire le prompt retour de ces citoyens, soutenant que le climat social et le dur labeur aux ÉtatsUnis leur deviendraient vite moralement insupportables. On leur pardonnerait leur erreur et on les recevrait aussi dignement que des enfants prodigues. H. Hurteau, officier d'immigration à Montréal en 1894, commente en ces termes le retour de 720 familles franco-américaines sur les terres qu'elles avaient abandonnées quelque temps auparavant, «dans l'espoir illusoire de mieux se tirer d'affaires dans les usines situées de l'autre côté de la frontière»:

[Their experiences] in recent times have tended to make these people realize their mistake as they never did before... They will find the plow and the harrow less irksome and more certain of reward for their labor than either lathe or loom. ${ }^{29}$

Les épreuves les avaient effectivement assagis, mais sans leur enlever l'espoir de pouvoir recommencer à neuf et réussir leur vie dans l'agriculture au Québec.

Lorsqu'il fut possible d'évaluer l'efficacité du rapatriement, les prévisions optimistes firent place à une appréciation plus réaliste des choses. Tout en se disant favorables à un prompt retour au Québec, la plupart des Franco-Américains restèrent aux États-Unis. Ceux qui revinrent de leur propre initiative, sans incitation de la part des «missionnaires-colonisateurs», déchantèrent rapidement, car ils trouvèrent des conditions économiques identiques à celles qui avaient provoqué leur exil, ou plus mauvaises encore. C'étaient souvent les fermetures d'usine qui provoquaient les retours, de sorte que les enfants prodigues retournaient au bercail dans la même misère qu'au départ. On a souvent vu dans la pauvreté des Franco-Américains un obstacle majeur à un rapatriement réussi. On craignait même que les conditions du retour n'aboutissent qu'à les décourager ${ }^{30}$. Il n'est donc pas surpre-

\footnotetext{
28 Voir, par exemple, les nombreux rapports des agents spéciaux du Ministère fédéral de l'Agriculture des années 1870. Canada: Sessional Papers, vol. VII à vol. XIII, 1874-1880 87.

Canada: Sessional Papers, 28, 9 (1895). Report of the Department of Interior (1894):

30 Québec: Documents de la Session, 1875, Vol. 5, Rapport du Commissaire de l'Agriculture pour $1872,347-350$.
} 
Origine géographique des colons ayant reçu des certificats au Lac-St-Jean, 1895-1904

\begin{tabular}{|c|c|c|c|c|c|c|c|c|c|c|c|c|c|c|c|c|c|c|}
\hline \multirow[t]{2}{*}{ Source } & \multicolumn{2}{|c|}{$1895-96$} & \multicolumn{2}{|c|}{1897} & \multicolumn{2}{|c|}{1898} & \multicolumn{2}{|c|}{1899} & \multicolumn{2}{|c|}{1900} & \multicolumn{2}{|c|}{1901} & \multicolumn{2}{|c|}{1902} & \multicolumn{2}{|c|}{1903} & \multicolumn{2}{|c|}{1904} \\
\hline & No & $\%$ & No & $\%$ & No & $\%$ & No & $\%$ & No & $\%$ & No & $\%$ & No & $\%$ & No & $\%$ & No & $\%$ \\
\hline Canada & & & & & & & & & & & & & & & & & & \\
\hline Québec & 982 & & 712 & & 638 & & 625 & & 755 & & 872 & & 1143 & & 1342 & & & \\
\hline Maritimes & 0 & & 0 & & 66 & & 128 & & 0 & & 0 & & 24 & & 23 & & & \\
\hline Ontario & 50 & & 15 & & 12 & & 14 & & 29 & & 28 & & 16 & & 31 & & & \\
\hline Total-Canada & 1032 & 63,8 & 727 & 57,2 & 716 & 53,9 & 767 & 45,8 & 784 & 41,8 & 900 & 42,8 & 1183 & 41,4 & 1396 & 46,3 & 1172 & 40,3 \\
\hline États-Unis & & & & & & & & & & & & & & & & & & \\
\hline $\begin{array}{l}\text { Nouvelle- } \\
\text { Angleterre }\end{array}$ & 373 & & 219 & & 411 & & 694 & & 845 & & 907 & & 1041 & & & & & \\
\hline Ailleurs & 107 & & 243 & & 201 & & 213 & & 142 & & 101 & & 87 & & & & & \\
\hline Total-E.-U. & 480 & 29,7 & 462 & 36,3 & 612 & 46,1 & 907 & 54,2 & 987 & 52,6 & 1008 & 47,9 & 1128 & 39,5 & 1114 & 37,0 & 1124 & 38,7 \\
\hline Europe & & & & & & & & & & & & & & & & & & \\
\hline Francophone & 105 & & 83 & & & & & & 98 & & 187 & & 214 & & 244 & & & \\
\hline Autres & & & & & & & & & 8 & & 8 & & 330 & & 260 & & & \\
\hline Total-Europe & 105 & 6,5 & 83 & 6,5 & 0 & 0 & 0 & 0 & 106 & 5,6 & 195 & 9,3 & 544 & 19,1 & 504 & 16,7 & 612 & 21,0 \\
\hline Total & 1617 & 100,0 & 1272 & 100,0 & 1328 & 100,0 & 1674 & 100,0 & 1877 & 100,0 & 2103 & 100,0 & 2855 & 100,0 & 3014 & 100,0 & 2908 & 100,0 \\
\hline
\end{tabular}


nant que ceux qui étaient revenus d'eux-mêmes réagirent rapidement lorsque les conditions économiques s'améliorèrent aux États-Unis. Plusieurs quittèrent le pays une seconde fois, réussissant même à convaincre des amis ou des membres de la famille qu'ils pouvaient connaître un sort meilleur dans les usines récemment réouvertes. Dans son rapport annuel de 1905, le docteur T.-A. Brisson de la Société générale de Colonisation et de Rapatriement émet cette réflexion:

When the crisis came in the United States three years ago, a larger number than one would believe of our emigrated compatriotes returned to Canada... In spite of attempts to retain them, a large number of those who had come back to us returned, or are now returning to foreign parts... as the opportunity occurs for them to take advantage of the reopening of the American manufacturies... The greater part of those who are leaving now, are those who had already been away, and it is feared that no efforts... can wholly prevent this. ${ }^{31}$

En fait, bon nombre de Franco-Américains traversèrent la frontière plusieurs fois durant leur vie active, ce qui leur valut le surnom de «coureurs de facterie» ${ }^{32}$. Ce comportement nomade ne manqua pas de donner très mauvaise réputation aux Franco-Américains auprès des colonisateurs du Québec et d'autres régions du Canada.

Quelques-uns des premiers promoteurs du rapatriement, qui avaient manifesté un optimisme sans borne, en vinrent à conseiller la prudence et la circonspection dans le choix des candidats. On admit peu à peu que le fait d'avoir subi une rapide prolétarisation dans les villes de la Nouvelle-Angleterre rendait les Canadiens français peu aptes ni même intéressés au travail ardu de défrichage qui était le lot dans les régions nouvellement colonisées. Le révérend Père $\mathrm{O}$. Corbeil, officier fédéral, remarqua après des visites à Lowell, Manchester et Nashua en 1895:

The French Canadians from these cities quickly loose (this is a personal observation) the skill of cultivating the land. They seem to lose, with their health, in manufacturing, that moral courage which made their fathers triumph (sic). ${ }^{33}$

31 Canada: Sessional Papers, 29, 10 (1896). Report of the Department of Interior (1895): 54-56. Il est d'ailleurs bien difficile d'obtenir des statistiques sûres sur le nombre de ces migrants «nomades». Ce va-et-vient s'opérait de façon assez rapide, selon les conditions économiques de part et d'autre de la frontière. Autre difficulté: comment faire la différence entre un émigrant et un rapatrié? On trouvera un bref commentaire sur la typologie des migrations dans Paul White et Robert Woods, (éd), The Geographical Impact of Migrations (Londres, New York, Longman, 1980), 18-19.

32 Cette désignation, fort appropriée, fut lancée par Pierre Anctil, un résident de Woonsocket, R.I., «La Franco-américanie ou le Québec d'en bas», Cahiers de Géographie du Québec, 23 (1979): 39-52. 53.

33 Canada: Sessional Papers, 29, 10 (1896). Report of Department of Interior, 13 (1895): 
En revanche, les Canadiens français installés dans le Midwest américain constituaient un groupe intéressant parce qu'ils vivaient déjà en milieu rural et travaillaient dans les secteurs des mines, de la forêt et de l'agriculture ${ }^{34}$. Les promoteurs du rapatriement estimaient que cette population constituait une bonne cible puisqu'elle n'était pas aussi urbanisée que celle de la Nouvelle-Angleterre et qu'elle avait encore des dispositions pour le travail de la terre. T.-A. Brisson recommanda l'envoi dans cette région de plusieurs officiers de rapatriement. Il remarqua qu'on devait faire une distinction entre les Franco-Américains de l'Est et ceux du Midwest:

Contrairement à ce que l'on observe dans les États de l'est où une forte proportion des Canadiens émigrés sont depuis longtemps livrés à l'industrie dans les manufactures et où il faut être très prudent dans le choix des colons qualifiés, on trouve dans le Michigan et autres États de l'ouest, une population de bûcherons et de fermiers que la destruction des forêts et la crise actuelle forcent à changer de résidence. Ce sont des colons armés de toutes pièces pour le défrichement de notre territoire qui demandent par milliers à tenter l'entreprise. On ne peut certes, désirer meilleure occasion pour opérer leur rapatriement. ${ }^{35}$

Les responsables du rapatriement ne partageaient pas tous cet avis. Seuls ceux qui avaient admis la faillite de certaines tentatives de rapatriement optaient pour une sélection basée sur le lieu de résidence et l'occupation du futur colon. Il s'en trouvait beaucoup d'autres à ne voir le rapatriement que d'un oeil patriotique ou politique et à rejeter toute distinction au sein de la population franco-américaine.

Les opinions divergeaient aussi sur des points d'ordre politique ou patriotique. Parfois, les Franco-Américains eux-mêmes donnèrent quelque opinion sur le rapatriement, mais leur message n'a pas toujours été bien interprété par les responsables du mouvement. Par exemple, l'officier de rapatriement, L.-A.-W. Proulx avance que:

Sauf quelques familles canadiennes qui préfèrent le travail dans les usines et les filatures à la culture des terres, et la résidence à la ville au séjour de la campagne, nos compatriotes sont très sympathiques à l'idée de revenir au Canada. ${ }^{36}$

Mais trois ans plus tard, un autre officier occupant le même poste énonce une opinion très différente: «Le rapatriement n'est pas populaire chez la majorité des émigrés et toutes démarches dans ce sens seraient inu-

34 Aidan McQuillan, «French Canadian Communities in the American Upper Midwest during the Nineteenth Century», Cahiers de Géographie du Québec, 23 (1979): 53-72.

35 Canada: Documents de la Session 1895, no 13, Rapport annuel de l'agent général de la Société Générale de Colonisation et de Rapatriement de Montréal pour 1894, 88.

36 Québec: Documents de la Session 1888. Rapport Général du Commissaire de l'Agriculture et de la Colonisation de la Province de Québec, 1888, 163. 
tiles dans les circonstances actuelles.» ${ }^{37}$ Pendant de nombreuses années encore ces divergences de vue continuèrent d'être alimentées, d'une part, par les constats d'échec d'une certaine forme de rapatriement et, d'autre part, par les espoirs naïfs de patriotes enflammés.

Certains faits particuliers indiquent que la Société de rapatriement se devait d'exercer du discernement quant au choix des colons. Au printemps et à l'été de 1897, un groupe de 170 Franco-Américains de Détroit revinrent au Québec, sous l'instigation de René Dupont, secrétaire et agent à l'étranger de la Société. En septembre de la même année, six des vingt-deux familles rapatriées décidèrent de retourner aux ÉtatsUnis, se plaignant que le gouvernement n'avait pas respecté ses engagements. Plusieurs journaux franco-américains se saisirent de l'affaire, ce qui ne contribua qu'à miner les efforts de la Société en faveur du rapatriement. Par la suite, les journaux publièrent une lettre du Consul général des États-Unis à Québec, qui appuyait la position des mécontents. Le directeur général du Lake St. John Railway, J.G. Scott, riposta que le consul avait probablement été induit en erreur et que le gouvernement avait tenu ses promesses. Il avait, en effet, gratuitement déménagé ces familles, avec armes et bagages, de Détroit à Québec. À partir de là, le Lake St. John Railway s'était chargé du transport. De plus, le gouvernement provincial avait alloué des terres à ces familles et leur avait offert un travail rémunéré dans la construction routière. Scott conclut que ceux qui étaient retournés à Détroit «were not suited to the hardships of the settlers' life». Il répondit dans le même sens à Sifton et à Laurier qui s'informaient de l'incident. En appendice à sa lettre à Laurier, Scott semble irrité au point de proposer pour l'avenir «...to let only those who wish to come from the United States who are prepared to pay their own expenses to Quebec, which will be a guarantee of their good faith and desire to remain here» ${ }^{38}$. Il s'agissait là d'une stratégie visant à éliminer les indésirables; elle semble, toutefois, n'avoir jamais été appliquée.

Les leçons tirées d'un quart de siècle de rapatriement et de la malheureuse aventure des immigrants de Détroit incitaient à l'adoption d'une politique plus rigoureuse de recrutement en favorisant les agriculteurs plutôt que les travailleurs urbanisés des villes de la Nouvelle-Angleterre. Encore ébranlé par l'histoire des gens de Détroit, Scott écrivit à Sifton: «...we intend to devote our efforts solely to settlers of the agricultural

37 Québec: Documents de la Session 1891, Rapport Général du Commissaire de l'Agriculture et de la Colonisation de la Province de Québec 1891, 56.

38 On fait état de l'incident de Détroit dans les documents suivants. Rapport dactylographié, Dupont à Scott, 6 décembre 1897, APC, RG-76, Immigration, vol. 129, file 28, 703, part I; lettre de Scott à Sifton, 16 mars 1898, ibid.; lettre de Scott à Laurier, 20 janvier 1898, APC, Laurier Papers, vol. 62, 19980; lettre de Scott à Laurier, 21 janvier 1898, ibid., 20027; lettre de Scott à Henry, 24 janvier 1898, ibid., vol. 63, 20132-20138. 
class.» ${ }^{39} \mathrm{Il}$ appert cependant que c'est précisément le contraire qui arriva, si on se fie aux chiffres du tableau qui précède. En 1897, les 219 colons rapatriés de la Nouvelle-Angleterre constituaient un peu moins de la moitié du nombre des Franco-Américains qui s'établirent au Lac-SaintJean. En 1902, ce nombre s'était gonflé à 1041 , soit $92 \%$ du total des immigrants. Le nombre de Franco-Américains venant des autres États diminua également. Somme toute, la Société réussit à attirer au LacSaint-Jean ceux-là mêmes qu'elle estimait les moins aptes à affronter les rigueurs de la colonisation.

Si la Société subit un échec relatif dans le Midwest, c'est sans doute que le rapatriement était alors un phénomène sujet à la concurrence $^{40}$. Il n'y avait pas que le Québec qui cherchait à attirer les Franco-Américains. Dans les années 1870, le Manitoba s'intéressait aussi à ce groupe, parce qu'à cette époque la survivance française y était menacée par l'arrivée récente d'un fort contingent de non francophones en provenance d'Ontario et des pays européens. Les efforts de rapatriement du Manitoba devinrent plus soutenus lorsque le Québec se mit à employer toutes sortes de manoeuvres pour décourager ses habitants d'émigrer dans l'Ouest canadien, même s'ils constituaient un excédent démographique destiné à émigrer de toute façon vers les ÉtatsUnis. Le rapatriement dans l'Ouest était appuyé par le gouvernement fédéral et les gouvernements provinciaux; mais dès le début, cet appui a été annulé par l'image négative que projetait l'Ouest, identifié à une Sibérie rude et inhospitalière, où les catholiques français n'étaient pas les bienvenus ${ }^{41}$. Il est un fait révélateur: les Franco-Manitobains finirent par arriver à la même conclusion que les responsables québécois du rapatriement, à savoir que le fermier du Midwest était le meilleur candidat pour la colonisation. Le Manitoba n'abandonna pas entièrement ses efforts en Nouvelle-Angleterre, mais il s'intéressa bien davantage au Midwest où, d'ailleurs, il enregistra ses plus fermes succès ${ }^{42}$. Après 1897, et malgré ses convictions, la Société fut de moins en moins active dans le Midwest et consacra toutes ses énergies aux villes manufacturières de la Nouvelle-Angleterre.

Tant au sein de la Société de rapatriement que chez l'élite francophone manitobaine, on estimait que le critère principal d'une éventuelle

39 Lettre de Scott à Sifton, 16 mars 1898, APC, RG-76, General Immigration Files, vol. 129, file 28703, part I.

40 Cet aspect de l'immigration est traité dans Robert LeBlanc, «Regional Competition for Franco-American Repatriates, 1870-1920», Quebec Studies, I (1983): 110-129.

41 Canada: Sessional Papers, 24 (1891). Report of Minister of Agriculture (1890): $173-$ 74; Canada: Sessional Papers, 26,8 (1893). Annual Report, Dept. of Interior, 13 (1892): 100.

${ }_{42}$ C'est le révérend Père J.-B. Morin qui mit au point la stratégie de recrutement auprès de la population agricole du Midwest; plus tard, Laurier la recommanda à Sifton. Voir APC, Laurier Papers, 67: 30488-89, lettre de Laurier à Sifton, 13 février 1899. Voir aussi Canada: Sessional Papers, 32,10. Report of the Dept. of Interior, 13 (1897): 103, Canada: Sessional Papers, 29. 10 (1896). Report of the Dept. of Interior, 13 (1895): 48-52, et APC RG-76, General Immigration Files, File 10063, vol. 94, lettre de R. Goulet Jr. à Sifton, 19 octobre 1900. 
immigration chez les Franco-Américains était la capacité du candidat à devenir un bon colon. Au Québec, on constata aussi que les caractéristiques qui faisaient d'un Franco-Américain de la Nouvelle-Angleterre un mauvais défricheur, faisaient de lui, en revanche, un excellent candidat pour le secteur manufacturier. Comme le fait remarquer un officier d'immigration de Montréal, la plupart de ces immigrants gardaient une préférence pour la vie urbaine ${ }^{43}$; ils conservaient de plus beaucoup d'intérêt pour le travail en usine. On pourrait donc conclure qu'un rapatriement valable aurait dû faire appel à des propositions plus alléchantes que l'allocation d'une terre vierge à défricher aux confins du Québec. Il aurait fallu une économie plus diversifiée ${ }^{44}$. En poussant le raisonnement plus loin, on pourrait avancer qu'une industrialisation accrue aurait enrayé l'émigration et que le problème de rapatriement n'aurait pas existé. Il est bien clair que la disparité économique entre le Québec et la Nouvelle-Angleterre, l'une stagnante et l'autre en plein essor, fut le problème fondamental qui rendit l'émigration attrayante et le rapatriement difficile. La véracité de cette remarque trouve confirmation au cours de la Première guerre mondiale: les Franco-Américains quittèrent massivement les filatures de la Nouvelle-Angleterre en butte à des problèmes économiques, pour aller travailler dans les villes du Québec ${ }^{45}$.

\section{Les Européens}

Les efforts déployés en Europe en faveur de la colonisation au Lac-Saint-Jean furent de courte durée et restèrent très restreints en comparaison de ce qu'ils furent ailleurs. Ces activités donnent tout de même une bonne idée des objectifs généraux et des méthodes de la Société. De plus, elles servirent auprès des responsables de la Société de guide d'évaluation de différents groupes migratoires. L'immigration dans la vallée du Saint-Laurent n'était pas chose nouvelle après 1760. La période située en 1820 et 1850 vit l'arrivée de milliers de Britanniques laquelle, ajoutée à l'immigration en provenance des États-Unis, créa des milieux à forte densité et dominance anglophones et finit par transformer Montréal en une ville cosmopolite où les francophones étaient en minorité. Après 1850, les Européens continuèrent d'arriver au Canada, mais peu s'établirent au Québec ${ }^{46}$. Les craintes suscitées par le Rapport Durham en 1840 se dissipèrent peu à peu, car l'immigration déclina, alors que le taux de croissance de la population canadienne-française se mainte-

43 Québec: Documents de la Session, 40,10 (1907). Rapport Général du Ministre de la Colonisation, des Mines et des Pêcheries (1906): 141-42.

44 Il s'agit d'une conclusion avancée par l'agent de colonisation de Matapédia, Québec: Documents de la Session, 34,10, Rapport Général du Ministre de la Colonisation des Mines et des Pêcheries, 124. Voir aussi Canada: Débats de la Chambre des Communes, 1908, 836.

45 Cette observation est basée sur des données de la Société de Colonisation de Montréal que nous étudions actuellement.

46 Norman MacDonald, Canada: Immigration and Colonization, 1841-1903 (Toronto, MacMillan of Canada, 1968), Chapter 6. 
nait, ce qui, en 1883, permit à sir Hector Langevin, alors ministre des Travaux publics, de rassurer en ces termes la Chambre des Communes: «...There is no danger about our [French Canadians] keeping our own ground in this country and extending our hold to any part of the Dominion to which we think proper to go (sic).» ${ }^{47}$ Cette impression de sécurité était loin d'être partagée par les Canadiens français du Québec; il est même probable qu'elle fut éphémère.

La capacité de survie des Canadiens français au Québec ou dans d'autres parties du Dominion était, bien entendu, reliée à divers facteurs, dont le plus important était leur force numérique. Les nationalistes s'inquiétaient de l'exode constant de citoyens québécois et de l'entrée de plus en plus importante d'immigrants européens, dont très peu de Français. Monsieur Tassé, député à Ottawa, essaya de démontrer la bonne foi des membres francophones du Parlement, faisant valoir qu'ils avaient appuyé l'immigration en dépit du fait que

We know very well... that every expenditure for foreign immigration purposes may reduce to some extent our influence in the community. We, the Frenchmen of Canada, draw no strength from that source. Old France does not send us any more of its blood... it seems to have none to spare. ${ }^{48}$

Vers la fin du dix-neuvième siècle, l'inquiétude relative à la survie de la culture française commença à s'intensifier.

La formation de la Société de rapatriement coïncida avec la politique agressive d'Ottawa en faveur de la colonisation dans l'Ouest, sous l'administration de Clifford Sifton. Cette politique exigeait que le gouvernement fédéral s'impliquât davantage dans le rapatriement, ce qui pouvait être considéré comme compatible avec les objectifs de la Société et ceux du gouvernement québécois. Ironiquement, le ministère fédéral de l'Immigration ne récolta au Québec que d'acerbes critiques, dictées tant par l'aveugle partisanerie politique que par l'authentique nationalisme ${ }^{49}$. Le Québec soutenait que la nouvelle politique d'immigration ne faisait que détourner, en faveur de l'Ouest, des fonds qui auraient pu être utilisés par les «vieilles» provinces pour mettre un frein à l'émigration et promouvoir le rapatriement dans l'Est. Les nationalistes accusaient également Ottawa de détourner vers l'Ouest des immigrants européens qui auraient pu choisir de s'établir dans l'Est. De plus, l'intérêt croissant de Sifton pour des colons possédant une expérience agricole aboutit finalement à un conflit ouvert entre le ministère de

47 Canada: Débats de la Chambre des Communes (1883): 894.

48 Ibid., 893.

49 On trouve un échantillon littéraire de ce nationalisme passionné dans la Minerve, décembre 1905. Laurier s'attendait à une opposition de la part des députés conservateurs du Québec à sa politique d'immigration, «In [their] hope of affecting our strength in the province.» Lettre de Laurier à Sifton, 13 février 1899, APC, Laurier Papers, 19: 30488-89. 
l'Immigration et la Société. Les officiers de rapatriement d'Ottawa concentrèrent leurs efforts de recrutement sur le prometteur Midwest américain, et ils dirigèrent leur récolte vers les Prairies. La Société protesta auprès d'Ottawa, soutenant que les Franco-Américains rapatriés étaient des candidats mieux adaptés au

Newly opened back country of... Quebec in preference to the North West Territories as they are naturally and more easily familiarized with the local conditions of the country and consequently are better fitted for the opening up of timber land instead of open prairie. ${ }^{50}$

Malgré de telles protestations, on continua à diriger les immigrants franco-américains et européens vers l'Ouest.

La politique d'Ottawa de venir chercher au Québec les «surplus sons of the older parishes» pour les diriger vers l'Ouest fut également perçue comme une pratique exécrable ${ }^{51}$. Le ministère de l'Immigration prétendit que ces gens auraient de toute façon quitté le Québec pour les États-Unis. Cette attitude d'Ottawa fut néanmoins considérée comme une atteinte directe aux efforts de la Société. René Dupont profita de la situation pour tenter d'obtenir de nouveaux appuis financiers:

We [in the Society] believe it is our duty to remark that Federal government has its paid agents working in our Province to draw away French Canadian settlers to the North West Territories, a work which is officially described as being so many new settlers: whilst we do similar work in our old settled parishes have not yet been successful in having our «settlers» recognized as such (for purposes of financial support). Notwithstanding, the gain is the same in both since if these settlers, gathered from the old parishes, did not take up new land in our [colonizing] regions they would be obliged to emigrate, which would be a loss to the country; so that in reality our work is as much immigration work as that of the agents working to obtain immigrants for the North West. ${ }^{52}$

Convaincu peut-être moins par l'argument que par les pressions de la Société, Laurier finit par lui accorder au printemps de 1901, une subvention de $3000,00 \$$ en plus des $8000,00 \$$ déjà versés annuellement à la Compagnie de chemin de fer du Lac-Saint-Jean ${ }^{53}$.

50 Requête adressée à tLaurier et à Sifton par les officiers de la Société du Lac Saint-Jean, 13 février 1900. APC, RG-76, Immigration, vol. 129, file 28703, part I. 199.

51 Canada: Sessional Papers, 34,10 (1900). Report of the Dept. of Interior, 13 (1899):

52 Rapport annuel de 1900. Société de Rapatriement et de Colonisation du Lac Saint-Jean, Québec, N. P., 1901,6.

53 Dès l'institution de la Société, ses cadres supérieurs, en particulier René Dupont, firent des représentations continuelles pour obtenir de l'argent, auprès de Laurier, de Sifton et de Pedley, le surintendant à l'immigration. Voir cette correspondance dans APC, Laurier Papers et APC, RG-76, Immigration, vol. 29, Archives de la Société du Lac Saint-Jean. Dans sa lettre annonçant l'octroi de $3000,00 \$$, Laurier avertit la Société qu'elle ne devait pas escompter le renouvellement de cet octroi. Il demanda également à la Société de réclamer de ses membres une plus forte participation financière. Il s'engageait, le cas échéant, à verser sa contribution personnelle. Lettre de Laurier à Dupont, 8 juin 1901, APC, Laurier Papers, (67): 56, 987. 
Le Québec, entre autres, s'élevait contre la pratique d'Ottawa d'encourager l'immigration sur une vaste échelle de paysans d'Europe centrale, la plupart d'origine slave. On se demandait pourquoi le gouvernement fédéral, faisait venir de si loin, à grands frais, des gens dont rien ne garantissait la loyauté future envers le pays, l'assimilation ou la permanence. Il y avait pourtant d'autres candidats intéressants au Canada même. D'autre part, raisonnait-on, si le gouvernement se donnait la peine de revigorer l'économie, l'émigration cesserait et les expatriés reviendraient au pays. Si Ottawa tenait à tout prix à l'immigration en provenance d'Europe, pourquoi, ajoutait-on, ne pas plutôt faire venir des francophones de France ou de Belgique, qui trouveraient au Québec un milieu plus propice à leur adaptation ${ }^{54}$ ?

Voilà donc dans quel cadre conflictuel s'exercèrent les activités de la Société en ce qui concerne l'immigration en provenance d'Europe. Le tableau indique qu'on rechercha particulièrement des candidats francophones, du moins au début. Les 188 immigrants en provenance de France, qui débarquèrent en 1896 et 1897 , furent probablement très bien accueillis, même si ce groupe ne représentait que $6 \%$ du total pour les deux années considérées. On ne sait pas si, à l'origine, ils furent approchés par les officiers fédéraux ou ceux de la Société. En dépit des effectifs minimes de francophones européens, certains nourrissaient encore des espoirs naïfs.

Il n'y a pas de doute que la persécution religieuse qui se poursuit actuellement en France nous sera très profitable et que nous sommes certains de ce chef d'obtenir une très bonne classe de colons, surtout au point de vue des moeurs et des idées. Car cette guerre se poursuit contre la partie la plus saine et la plus religieuse de la vieille France. ${ }^{55}$

En 1902, la Société avait pu se gagner l'appui du délégué fédéral d'immigration à Paris. Par la suite, elle y envoya son propre délégué, dans l'espoir que les politiques anti-cléricales du gouvernement WaldeckRousseau, seraient propices au recrutement ${ }^{56}$. Il est possible que la persécution religieuse en France et les efforts de la Société aient pu favoriser l'immigration au Canada après 1899. Toutefois, il semble que

54 Québec: Documents de la Session, 37 (1904): 7. Rapport Général du Ministre de la Colonisation et des Travaux Publics pour 1903, 113-114: Québec:Documents de la Session, 1906, 39: 7, Rapport Général du Ministre de la Colonisation et des Travaux Publics pour 1905, 120122.

Société de Rapatriement et de Colonisation du Lac Saint-Jean. Rapport annuel pour l'année 1903, Québec: N. P., 1904, 10.

56 Lettre de Dupont à Smart, 15 mai 1902, APC, RG-76, Immigration, vol. 129, file 28703, part 2. Voir aussi Guy Laperrière, «Persécution et exil»: la venue au Québec des congrégations françaises, 1900-1914», Revue d'histoire de l'Amérique française, 36,3 (décembre 1982): 389411 . 
la plupart des nouveaux venus choisirent plutôt de s'établir dans l'Ouest ${ }^{57}$.

Le nombre des colons recrutés dans les pays non-francophones d'Europe n'est pas plus significatif que ceux en provenance de France ou de Belgique; cela donne tout de même une idée de la nature compétitive de l'immigration. La plupart venaient d'Europe du Nord, particulièrement de Scandinavie. Informée de l'intérêt manifesté par des Finlandais pour le Canada, la Société pria le ministère de l'Immigration de faire le nécessaire pour que quelques-uns de ces candidats soient dirigés vers le Lac-Saint-Jean, faisant valoir que ces gens «are accustomed to a northern climate, they will prove very suitable settlers» ${ }^{58}$. Lors d'un voyage en Europe, au début de l'hiver 1901-1902, René Dupont réussit à convaincre certains Finlandais de s'établir au Québec et le représentant d'Ottawa à Londres eut la surprise d'apprendre le départ d'une centaine de Finlandais pour Saint-Jean, NouveauBrunswick, en mars $1902^{59}$. Il informa Pedley que la destination de ces immigrants était le Québec mais que

If they can be persuaded to go through [to the West], I fancy you will find that this is the beginning of a considerable movement from Finland. I am sure you will understand the importance of having steps taken to secure them good locations in the Western country.

Pedley partageait cet avis mais dut tout de même admettre qu'il s'agissait d'une «affaire délicate». Il demanda donc au représentant fédéral à Saint-Jean, Nouveau-Brunswick, monsieur J. V. Lantanum «to quietly ascertain how the matter stands and do what is best in your judgment, after meeting these people and discussing the matter with them». Lantanum lui fit part de son impuissance à intervenir, étant donné que le capitaine Labelle, représentant du gouvernement québécois, avait déjà pris les dispositions pour le transfert des Finlandais vers le Lac-SaintJean. Lantanum rapporta que, même après le départ des Finlandais, il essaya de convaincre leurs chefs de s'établir plutôt dans l'Ouest. Ce fut en vain, mais il obtint tout de même de ces derniers l'assurance que si les choses ne les satisfaisaient pas au Québec, ils ne se gêneraient pas pour s'en aller rapidement ailleurs. Entre-temps, la presse québécoise annonçait avec enthousiasme la venue de ce premier groupe de Finlan-

\footnotetext{
57 Québec: Documents de la Session 1906, 39, 7. Rapport Général du Ministre de la Colonisation et des Travaux Publics pour 1905, 120-122.

58 Lettre de Scott à Sifton, 20 avril 1899, APC, RG-76, Immigration, vol. 129, file 28703, part 1, et lettre de Jules Tessier (Président de la Société) à Laurier, 25 février 1902, APC, Laurier Papers (67): 63, 119-163.

59 Cet épisode de l'immigration des Finlandais au Lac-Saint-Jean est basé sur la correspondance entre Pedley, surintendant à l'immigration, et les agents J. V. Lantanum (St-Jean, N. B.), P. Doyle (Ville de Québec) et W. D. Preston (Londres). APC, RG-76, Immigration, vol. 129, file 28703 , part 2 .
} 
dais et laissait espérer d'autres arrivées. Quelques autres familles vinrent, en effet, en 1903. Dupont assura Ottawa que les Finlandais s'acclimateraient aisément au milieu québécois; il les voyait même comme «a people [who] resemble our own [French] Canadians in appearance more than any other foreigners» ${ }^{60}$. Toutefois, la plupart des Finlandais quittèrent le Québec après quelques mois.

Ni le Québec, ni la Société ne manifestèrent d'appréhension de voir arriver dans la province des «étrangers». D'abord, il s'agissait là d'une politique délibérée; de plus, on s'attendait à ce que ces immigrants contribuent par leur travail à l'évolution économique de la région, ce qui, somme toute, était l'objectif principal de la Compagnie de chemins de fer. En l'occurrence, les objectifs nationalistes de la Société se faisaient extrêmement discrets, espérant qu'un si petit groupe serait rapidement assimilé.

Même si ce genre de rationalisation semblait acceptable pour le Québec, cela n'empêchait pas certains de s'élever contre toute immigration «étrangère» dans d'autres parties du pays. Peu de temps après que Jules Tessier, président de la Société, eût, avec une évidente satisfaction, informé Laurier de l'arrivée des Finlandais, Israël Tarte, ardent nationaliste et directeur de la Société, protesta avec véhémence contre l'arrivée au Manitoba et dans les Territoires du Nord-Ouest d'étrangers «dont une bonne partie ne vaut pas grand'chose» ${ }^{61}$. Quoique discrète, l'opposition à l'immigration étrangère fut toujours présente. Le curé $\mathrm{H}$. Paradis de Roberval en a laissé une preuve: «...j'ai toujours été absolument contre l'immigration d'étrangers dans la province de Québec et surtout au Lac Saint-Jean.... ${ }^{62}$

Quelques années après le départ des Finlandais du Québec, le Père E. Gauthier, officier fédéral de recrutement d'immigrants, avouait à un auditoire de Manchester, New Hampshire, que ce que le Canada avait gagné en nombre par l'immigration étrangère, il l'avait perdu en qualité:

Il y a six ou sept ans on a fait venir à la Baie de Ha Ha 500 Finlandais peuplade du nord de la Russie. Sans compter qu'ils parlaient un language que personne ne comprenait, sans parler de leur religion qui n'était en aucune façon conforme à la nôtre, nous pouvons dire qu'ils ne valaient pas, même pour le travail manuel, 200 de nos Canadiens. Le résultat, c'est qu'au bout d'un an, il n'en restait pas un seul. ${ }^{63}$

60 Lettre de Dupont à Smart, 8 avril 1902, Ibid.

61 Lettre de Tessier à Laurier, 25 février 1902, APC, Laurier Papers (67): 63, 119-163, et lettre de Tarte à Laurier, 16 juin 1903, Ibid., 65654.

62 Lettre de Paradis à Dupont, 25 octobre 1906, Ibid., 11, 345-11, 346.

63 Tiré de l'Avenir National (Manchester, N. H.), 27 février 1908. 
Gauthier assura donc son auditoire que le Canada s'intéressait maintenant davantage au million et demi de Canadiens français émigrés aux États-Unis qu'à des étrangers de ce calibre.

\section{Les Canadiens français}

La zone migratoire la plus importante était, bien sûr, celle des vieilles paroisses des terres basses du Saint-Laurent. C'est là que se trouvait un excédent démographique sans perspective d'avenir dans sa région d'origine. On se devait de lui offrir la possibilité de s'établir ailleurs au Québec, pour éviter l'émigration. Ce groupe représentait, en fait, la raison d'être fondamentale du mouvement de colonisation. La réussite de celui-ci aurait rendu le rapatriement inutile. Malheureusement, la situation se détériora à tel point que des milliers de Canadiens français quittèrent le pays, exode qui menaçait de plus en plus de miner le pouvoir politique des francophones et constituait un vrai cassetête pour les leaders du mouvement de colonisation. Certains étaient d'avis que les faibles ressources dont disposaient les gouvernements devaient d'abord être dépensées au Québec pour consolider sa population plutôt que pour rapatrier des défricheurs de valeur douteuse ${ }^{64}$.

Dès le départ, la Société rejeta ce point de vue. Son nom même de Société de colonisation et de rapatriement indique clairement qu'elle avait l'intention d'exercer ses activités des deux côtés de la frontière. Malgré ses déboires du début avec les Franco-Américains, elle n'en continua pas moins à dépenser des sommes substantielles dans les villes manufacturières de la Nouvelle-Angleterre, effort, il faut le dire, qui donna des résultats satisfaisants, à en juger par le nombre de colons franco-américains recrutés. On ne négligea pas, toutefois, les intéressantes zones migratoires du Québec même.

Quant à la question d'estimer quelle était la zone migratoire la plus propice et quel individu représentait le colon idéal, la plupart des avis rejoignaient celui du curé de Roberval: «Il faut des gens du pays [au Lac Saint-Jean]; des Canadiens français que nous trouverons dans les grandes et belles paroisses des comtés environnant Québec, Montréal et Trois-Rivières.» ${ }^{65}$ Dupont avait déjà donné son opinion sur le sujet devant une commission d'enquête en 1903. Il avait identifié trois groupes auxquels s'adressait l'activité de la Société: les habitants des vieilles paroisses, les Franco-Américains et les Européens. Quand on lui demanda lequel des groupes était le plus approprié, il n'hésita pas à répondre:

\footnotetext{
64 Canada: Sessional Papers, 29, 10 (1896). Report of Department of Interior, 13 (1895): 54-56. 346 .

65 Lettre de Paradis à. Dupont, 25 octobre 1906, APC, Laurier Papers, (67): 11, 345-11,
} 
Ce sont nos canadiens-français; les étrangers ne sont pas généralement défricheurs; ceux qui viennent d'Europe ne connaissent pas du tout la colonisation ou plutôt le défrichement. Il convient ici de faire une distinction entre les divers colons qui nous arrivent; il y a le colon défricheur et le colon cultivateur; nos canadiens colons défricheurs ont ordinairement très peu d'argent à leur arrivée, les Européens et les rapatriés sont généralement des colons cultivateurs. Ils arrivent généralement avec certains moyens et font avancer leurs lots par les défricheurs et le plus souvent, les premiers achètent des lots en partie défrichés des derniers. (sic) ${ }^{66}$

La supériorité présumée des gens du pays comme défricheurs ne fut jamais mise en doute. Le tableau révèle cependant que le pourcentage de colons fournis par le Québec diminua d'année en année par rapport au pourcentage total. De plus, il est probable qu'une portion de ces gens ne répondait même pas à la définition de «gens du pays» parce qu'ils émanaient d'un milieu urbain ${ }^{67}$.

Peu de certificats de colonisation furent émis en faveur de francophones hors Québec. On ne trouve pas non plus de déclaration d'intention de la Société d'attirer des Franco-Ontariens et des Acadiens. Pourtant on fit de la publicité dans les Maritimes. C'est ainsi que les Acadiens de Chéticamp quittèrent leur village de pêche du Cap-Breton pour devenir des agriculteurs au Nord-Est québécois. L'arrivée au Québec de 200 Acadiens, en 1898-1899, laissa croire que d'autres suivraient ${ }^{68}$. Mais cela ne se produisit pas. Nous en concluons que leur établissement au Québec ne fut pas très réussi, ce qui probablement découragea toute migration additionnelle. Il se peut même que beaucoup retournèrent à Chéticamp ${ }^{69}$.

\section{Dissolution de la Société}

En janvier 1907, la Société fut dissoute et ses opérations prises en charge par le ministère fédéral de l'Immigration. Comme lors de sa

\footnotetext{
66 Enquête faite par la Commission de Colonisation dans la région du Lac Saint-Jean à la demande de la Société de Rapatriement et de Colonisation du Lac Saint-Jean, du 14 au 25 décembre 1903 (dactylographié). Fonds de la Société historique du Saguenay, Dossier 1256, pièce 1, 2.

67 Les rapports annuels de la Société (à partir desquels sont compilées les données du Tableau 1), fournissent des renseignements détaillés sur les lieux d'origine des colons, de part et d'autre de la frontière. Du total québécois, $27 \%$ originent de Montréal, de Québec et de la Mauricie; $17 \%$ de Charlevoix et de Montmorency, comtés où la population occupait des terres agricoles marginales, c'est-à-dire très différentes de ce qu'on appelait «les vieilles paroisses des terres basses». Les migrants en provenance des comtés au nord-est de Trois-Rivières comptent pour $46 \%$ du total de la province. C'est cette région qui constitua la source privilégiée des toutes premières migrations au Lac-Saint-Jean. Voir Normand Séguin, Agriculture et Colonisation au Québec: Aspects historiques (Montréal, Boréal Express, 1980), 162.

68 Société de Rapatriement et de Colonisation du Lac Saint-Jean, Rapport Annuel pour l'année 1898. Québec, N. P., 1899.

69 Rév. Père Anselme Chiasson, Cheticamp: histoire et traditions acadiennes (Moncton, Éditions des Aboiteaux, 1961), 36.
} 
fondation, ce transfert s'opéra dans un climat d'amères discordes. Si l'on étudie plus à fond les causes de cette fin prématurée, on constate d'abord que la colonisation fut, avant tout, une entité essentiellement idéologique. De plus, on peut saisir toute l'ampleur du conflit qui opposa la Société au gouvernement fédéral, malgré des objectifs en apparence compatibles.

Nous avons déjà fait ressortir les conflits qui pouvaient naître des objectifs des deux parties. Ce fut, en particulier, l'ardeur d'Ottawa à l'égard du peuplement des Prairies qui mit le feu aux poudres. On accusa Ottawa de venir piller les vieilles paroisses du Québec en faveur de la colonisation dans l'Ouest, stratégie qu'appuyait, bien entendu, l'élite francophone de l'Ouest. On l'accusa en plus de détourner vers l'Ouest l'immigration en provenance d'Europe, aux dépens des provinces de l'Est. On vilipenda aussi le gouvernement central pour avoir, semblet-il, accordé d'extravagantes subventions aux chemins de fer de l'Ouest tout en refusant sa générosité à ceux de l'Est. Ce favoritisme se manifestait non seulement dans les politiques de peuplement, mais aussi dans celles de recrutement des colons:

It is remarkable fact that the Canadian government has its immigration agents in Scotland, in Ireland, in England, in Belgium, in Finland even, as well as the Western States, while by a singular anomaly it has not a simple (sic) agent at work in the Eastern States. ${ }^{7}$

En dépit des efforts sincères de Laurier pour donner quelque satisfaction aux nationalistes et aux dirigeants de la Société, les plaintes, parfois injustifiées, continuèrent d'affluer à Ottawa.

Comme premier francophone à devenir Premier ministre du Canada, comme libéral souvent opposé aux nationalistes québécois, Laurier se voyait inévitablement impliqué dans ce dossier. C'est certainement grâce à son appui moral et financier que la Société put voir le jour. Il était constamment harcelé par ses collègues et ses amis qui faisaient appel à son patriotisme de Canadien français, quand ils ne le menaçaient pas tout simplement de représailles politiques. Il invitait tous les ans un groupe de délégués de la Société à venir lui présenter ses demandes. Il était donc tout à fait normal qu'il eût à jouer un rôle déterminant dans la dissolution de la Société.

Les premières difficultés surgirent dès le départ, lorsque Laurier fit savoir comment il percevait le rôle d'une société de colonisation et les attributs de ses membres. Une correspondance acrimonieuse avec Israël Tarte, au cours de l'été 1902, révèle sa position et celle de la

70 Société de Rapatriement et de Colonisation du Lac Saint-Jean. Rapport Annuel pour l'année 1900, 5. 
Société. Dans une première lettre, Tarte demandait à Laurier son appui pour obtenir une augmentation de la subvention fédérale en faveur de la Société:

Ne pourriez-vous pas demander à M. Sifton de ne pas tout jeter dans l'Ouest. Il me semble que nous ne serons pas taxés d'extravagance en réclamant la modeste petite somme de \$6000 quand nous dépensons environ un demi-million par an, pour peupler l'ouest.

Laurier rappela simplement à son correspondant que son gouvernement avait promis d'augmenter la subvention à la condition que la Société augmentât le montant des cotisations des membres. Un total des cotisations de 150,00\$ lui semblait ridicule. Quant à l'accusation de favoriser l'Ouest, il déclara: «Il y a toute la différence du monde entre les gens du Nord-Ouest et les membres de la Société de colonisation. La Société de Colonisation est une société philanthropique dont l'objet est excellent.» ${ }^{72}$ Tarte n'épousait pas ce point de vue. Il répliqua que la seule différence, à son avis, était que la Société opérait dans une région canadienne-française et que, par conséquent, elle était devenue un objet de discrimination. Laurier insista sur la distinction déjà relevée plus haut, indiquant que le gouvernement fédéral avait à jouer un rôle plus large que celui de la Société. Voilà pourquoi il s'impliquait dans la colonisation de l'Ouest. Il ajouta que la colonisation dans l'Est relevait davantage des gouvernements provinciaux lorsque les sociétés locales de colonisation étaient en difficulté. Malgré l'optique du Premier ministre, le ministère de l'Immigration n'en continua pas moins à appuyer la colonisation au Québec, sur une échelle réduite, il est vrai.

On fit tous les efforts pour empêcher l'effondrement de la Société jusqu'en $1906^{73}$, plus précisément jusqu'au jour où J. Girard, député de Chicoutimi à Ottawa, et jusqu'ici l'un des plus ardents partisans de la Société, cessa de lui donner son appui. Dans une lettre adressée à Laurier, Girard expliqua pourquoi la Société avait perdu sa confiance, celle du clergé et celle de l'élite du Lac-Saint-Jean. Selon lui, la Société s'était rendue coupable de falsification de documents relatifs au tableau des membres et aux cotisations; de propagande trompeuse à l'égard du recrutement, ce qui avait eu pour effet de décourager les colons de

71 Lettre de Tarte à Laurier, 6 juin 1902, APC, Laurier Papers (67): 65652

72 Lettre de Laurier à Tarte, 2 juillet 1902, APC, Laurier Papers (67): 65655. La conception que se faisait Laurier d'une société de colonisation ressemble fort à celle avancée par $\mathrm{H}$. Langevin au cours d'un débat à la Chambre des communes, vingt ans auparavant: «I do not say that something more cannot be done by the [federal] Governments [in support of repatriation]; but a good deal can be done by these people themselves, and also by those colonization societies, which are really societies of the people because they come into existence by subscriptions from the people». Voir Débats, Chambre des communes, 1883, 895.

${ }_{73}$ Voir, par exemple, Rossel Vien, Histoire de Roberval, coeur du Lac Saint-Jean (Chicoutimi, La Société Historique du Saguenay, 1955), 183. 
fausse représentation en s'attribuant le crédit de l'augmentation de la population régionale en 1901; et enfin de mauvaise administration et de détournement de fonds. En conclusion, il ajoutait:

C'est pourquoi il est grand temps que la direction de l'avenir de mon district soit remis comme autrefois entre les mains du gouvernement et du public intéressé et résidant, vu que la direction étrangère, telle que nous l'avons par l'invasion de cette société fait plus de mal que de bien et compromet la position du gouvernement dans l'opinion publique. ${ }^{74}$

Bien entendu, les administrateurs de la Société protestèrent de leur innocence et accusèrent Girard de partisanerie malicieuse. Cela ne réussit pas à atténuer les pressions qui s'exerçaient sur la Société, et le 31 décembre 1906, celle-ci résolut de se dissoudre en faveur du gouvernement fédéral ${ }^{75}$.

\section{Conclusion}

Les basses terres du Lac-Saint-Jean n'étaient qu'une partie de la «terre promise», un des décors du grand théâtre de la colonisation des terres reculées du Québec ${ }^{76}$. Le développement de cette région fut intimement lié à la colonisation et au rapatriement, deux mouvements complémentaires qui ont préoccupé l'élite du Québec pendant près de cent ans, en réplique à l'exode massif d'un million de Canadiens français aux États-Unis entre 1840 et 1940. Si tout avait fonctionné selon les prévisions, la situation démolinguistique du Canada et du Québec eût été tout autre aujourd'hui ${ }^{77}$. Malheureusement, il était illusoire de vouloir retenir sur place ou de rapatrier des Canadiens français: l'émigration était motivée par des besoins économiques qu'on ne pouvait atténuer par l'expansion agricole sur des terres improductives. Les appels au patriotisme tombèrent dans des oreilles de sourds. Les excédents démographiques du Québec s'expatrièrent parce que l'agriculture était sans avenir; parce que l'économie régionale pataugeait dans de vieilles traditions; parce que les rênes de cette économie étaient tenues par une élite canadienne-française conservatrice, elle-même manipulée par une oligarchie étrangère, parfaitement insensible aux idéaux nationalistes des Québécois. Certes, quelques francophones quittèrent les villes de la Nouvelle-Angleterre en période de récession, pour revenir au Québec, mais leur séjour y fut de courte durée. Beaucoup reprirent le che-

\footnotetext{
74 Lettre de Girard à Laurier, 10 juillet 1906. APC, Laurier Papers (67): 112003-112006.

75 Lettre de Dupont à Laurier, 2 janvier 1907, APC, Laurier Papers (67): 117787-117788.

76 Péribonka, situé sur la rive nord du lac Saint-Jean, est la scène du roman «rural» le plus célèbre du Québec, Maria Chapdelaine, de Louis Hémon, dont on a tiré un long métrage en 1983.

77 Pour une analyse récente des aspects historiques et une perspective de la question, voir Jean Lachapelle et Jacques Henripin, The Demolinguistic Situation in Canada (Montreal, The Institute for Research on Public Policy, 1982).
} 
min vers le sud, souvent en compagnie d'autres Québécois, amenés, eux aussi, à fuir une économie stagnante.

La responsabilité de promouvoir la colonisation et le rapatriement fut, le plus souvent, assumée par les membres du clergé les plus ardents et les sociétés régionales de colonisation dirigées par l'élite locale. Quant au gouvernement provincial québécois, il n'y joua qu'un rôle occasionnel, souvent à contre-coeur, parce que les conservateurs de l'Assemblée législative le poussaient au pied du mur ${ }^{78}$. Pour sa part, le gouvernement fédéral s'y impliqua activement, quoique de manière intermittente. Les périodes d'ardeur ou de tiédeur étaient déterminées, soit par le désir de manifester la générosité du parti au pouvoir ou de mettre en pratique la politique d'encouragement de l'immigration, soit celui de restreindre les dépenses budgétaires. Tous s'entendaient sur les objectifs. Il en fut tout autrement quant aux modalités de réalisation. Des conflits surgirent entre diverses sociétés régionales de colonisation à la recherche de francophones. Cela nous permet de mieux comprendre l'aspect concurrentiel de l'immigration francophone, de nous faire une meilleure idée de l'ampleur de la dispersion de petits groupes de francophones sur le vaste territoire d'Amérique du Nord, et donc de saisir les raisons profondes de la peur de l'assimilation. Il n'est pas difficile de comprendre que le succès d'une société de colonisation signait presque inévitablement l'échec d'une autre. Bien d'autres conflits surgirent, dont l'objet était l'allocation et la répartition des octrois et leurs modes d'utilisation par ceux qui en bénéficiaient.

Si l'on observe maintenant les activités de la Compagnie de chemin de fer du Lac-Saint-Jean, non seulement on y trouve la confirmation des conflits rapportés plus haut, mais on constate aussi qu'aucun colon francophone n'accepta les rigueurs de la colonisation sans se plaindre des dures conditions de travail et des maigres résultats de son labeur. Les dirigeants de la Société durent admettre, comme bien d'autres auparavant, qu'il fallait exercer le plus grand discernement dans le choix des défricheurs et que, tout compte fait, les meilleurs colons se trouvaient au Québec même, au sein des paroisses rurales. Les FrancoAméricains de la Nouvelle-Angleterre s'avérèrent des candidats beaucoup moins intéressants parce que trop urbanisés. Malgré tout, la Société déploya des énergies considérables sur ce territoire. Le rapatriement de quelques milliers de colons représente un faux taux de réussite exagéré cependant, puisque la plupart reprirent le chemin des États-Unis ${ }^{79}$. Les quelques efforts de la Société en Europe donnèrent très peu de résultats

78 Marcel Hamelin, Les premières années du parlementarisme québécois, 1867-1878. Coll. «Cahiers d'histoire de l'Université Laval», no 19 (Québec, Les Presses de l'Université Laval, 1976), 181-183.

' 79 Société de Rapatriement et de Colonisation du Lac Saint-Jean. Rapport Annuel pour l'année 1903, Québec, N. P., 1904, 4. 
concrets; ils déclenchèrent, tout de même, une prise de conscience qui favorisa la «rationalisation» de ce type d'immigration au Québec. La plupart des nouveaux colons, quelle que fut leur origine, redevinrent des émigrants, après une décourageante expérience de vie agricole. Pour assurer au Saguenay l'essor économique, il fallut ultérieurement développer les ressources forestières et énergétiques et non pas les possibilités agricoles. La clef du problème résidait donc dans l'économie. Sans de meilleures perspectives au Saguenay, les Franco-Américains rapatriés, les Européens, et les «gens du pays» devaient chercher ailleurs la prospérité. 